\title{
Introduction and annealing of primary defects in proton-bombarded n-GaN
}

\author{
Matthias Schmidt, ${ }^{*}$ Hannes de Meyer, Pieter Johan Janse van Rensburg, Walter Ernst Meyer, and \\ Francois Danie Auret \\ Department of Physics, University of Pretoria, Private bag X20, 0028 Hatfield, South Africa
}

${ }^{*}$ Corresponding author: e-mail schmidt.matthias@matschmi.de, Phone: +27 124202684, Fax: +27 123625288

\begin{abstract}
We report on in situ space charge spectroscopy measure-ments on low-temperature $1.6 \mathrm{MeV}$ proton-bombarded n-type gallium nitride thin film samples. The scope of this study was to investigate the introduction and annealing dynamics of radiation-induced lattice damage. Using optical excitation allowed for the detection of electronic defect states in the entire GaN bandgap and to detect unstable primary defects that would have been invisible in thermal space charge spectroscopic measurements. The introduction of compensating acceptor-like primary defects by the bombardment was observed and manifested as a decrease in the sample capacitance. After the bombardment the concentrations of deep-levels and acceptor states were monitored by deep-level transient spectroscopy and photo-capacitance measurements while the temperature was increased. It was found that annealing and reactions of primary bombardment-induced defects occurs even below room-temperature which might account for the radiation-hardness of GaN.
\end{abstract}

Keywords capacitance-voltage spectroscopy, deep levels, defects, DLTS, GaN, proton irradiation

\section{Introduction}

It is well known that the opto-electronic properties of semiconductors strongly depend on crystal imperfections such as impurities or lattice defects. In essence this is due to electronic states that are often introduced into the semiconductor bandgap by these entities. Due to thermodynamic reasons a certain number of lattice defects is always present in a crystal at thermal equilibrium. In addition, lattice defects can be introduced when the semiconductor is exposed to high energy particle radiation as found in the nuclear industry or in outer space.

The wide-bandgap $(\approx 3.4 \mathrm{eV})$ semiconductor gallium nitride $(\mathrm{GaN})$ and related compounds are commonly used materials in UV opto-electronics and white light emitting diodes [1]. The radiation hardness of $\mathrm{GaN}$ has previously been investigated in $\mathrm{GaN}$ thin films [2-7], single crystals [8], nanowires [9], and devices [10,11]. An overview on radiation effects in $\mathrm{GaN}$ can be found in Ref. [12]. Thereby GaN was found to be far more radiation-tolerant than for instance GaAs. On the one hand this can be explained by the stiff $\mathrm{GaN}$ lattice for which the displacement energies are 19 and $22 \mathrm{eV}$ for gallium and nitrogen, respectively [13,14]. For comparison the threshold for lattice damage in GaAs is only $10 \mathrm{eV}$ [15]. On the other hand an efficient annealing of radiation-induced defects even below room-temperature could also account for the previously observed radiation-tolerance. To answer this question, it is necessary to conduct high-energy particle irradiation experiments at cryogenic temperatures and characterise the induced defects in situ in the same setup without exposing the sample to room-temperature in between. This is the only possibility to avoid annealing or defect reactions of primary defects that occur even at low temperatures. Most of the studies on the radiation hardness of $\mathrm{GaN}$ and studies on irradiation-induced defects were conducted such that the sample was exposed to room-temperature between irradiation and characterisation experiments. Therefore, the literature on in situ studies is scarce. Optically detected electron paramagnetic resonance (ODEPR) studies on lowtemperature electron-irradiated GaN samples were carried out by Chow et al. [3, 4]. They investigated the interstitial gallium and the lattice damage in the gallium sublattice. To best of our knowledge there are no reports on in situ irradiation 
experiments using space charge spectroscopy to characterise the defects.

In this work, we investigated the introduction and annealing dynamics of (primary) lattice defects in protonbombarded GaN by means of space charge spectroscopy. The advantage of space charge spectroscopy compared to other methods is its high sensitivity to low concentrations $\left(<10^{15} \mathrm{~cm}^{-3}\right)$ of electronic defect states. Experiments of this type date back to the 1970s when Lang et al. $[16,17]$ investigated the annealing of deep levels in electron-irradiated gallium arsenide at elevated temperatures by deep level transient spectroscopy (DLTS). Thereby the main difficulty is the breakdown of the validity of the Born-Oppenheimer approximation in which it is assumed that electrons can be in free or bound states whereas the ion cores can oscillate but are bound to their lattice sites. In other words in space charge spectroscopic measurements one assumes the dynamics of the crystal to be in the electron system and the core system to be static. However, after particle-bombardment a crystal is in non-equilibrium and annealing processes and defect reactions occur which means that ion cores change lattice sites.

Obviously the dynamics in both, core and electron system, is temperature $T$ activated. If the lattice is at thermal equilibrium and the defect annealing occurs due to diffusion, the annealing rate of the defect (core system) is described by a Boltzmann function [21]

$$
e_{\mathrm{ann}} \propto \exp \left(-\frac{E_{\mathrm{ann}}}{k_{\mathrm{B}} T}\right)
$$

On the other hand, the rate at which a defect-bound electron is thermally emitted into the conduction band (CB) is also temperature activated [18]

$$
e_{\mathrm{n}}^{\text {th }} \propto T^{2} \exp \left(-\frac{E_{\mathrm{a}}}{k_{\mathrm{B}} T}\right) .
$$

$E_{\text {ann }}$ and $E_{\text {a }}$ denote the activation enthalpies for the annealing of the defect and its thermal ionisation, respectively, $k_{\mathrm{B}}$ is Boltzmann's constant. Please note that $E_{\text {ann }}$ [16] and $E_{\mathrm{a}}[19,20]$ can depend on the net doping density $N_{\text {net }}$ of the sample or the concentration of the defect itself. In many cases $E_{\text {ann }}$ is furthermore found to be recombination-enhanced and does therefore depend on the biasing conditions of the sample [17,21].

Defects that are considered to be stable, meaning they do not significantly anneal during the measurements, exhibit annealing rates $e_{\text {ann }} \ll 10^{-4} \mathrm{~Hz}$ for all temperatures that the sample undergoes. In turn this means that $E_{\text {ann }} \gg 1 \mathrm{eV}$. On the other hand electronic defect states can only be conveniently measured by thermal space charge spectroscopy if the thermal electron emission rate is $0.01 \mathrm{~Hz}<e_{\mathrm{n}}^{\text {th }}<1 \mathrm{MHz}$. For unstable defects the situation is depicted in Fig. 1: Assume the activation enthalpy for thermal ionisation of the electronic state of the defect is $E_{\mathrm{a}}=300 \mathrm{meV}$. For a typical electron capture cross-section of $\sigma_{n} \approx 10^{-15} \mathrm{~cm}^{2}, e_{\mathrm{n}}^{\text {th }}(T)$

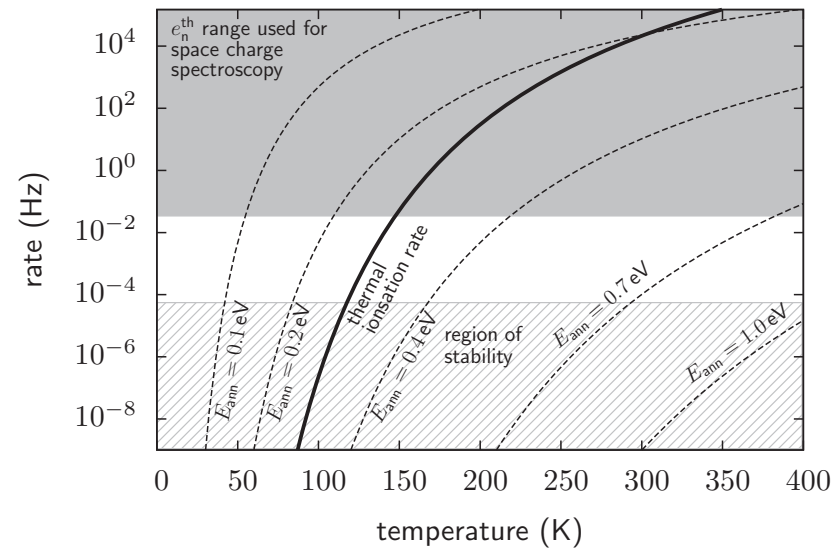

Figure 1 Limits of thermal space charge spectroscopic methods for the investigation of electronic states of unstable lattice defects. The dashed lines are the annealing rates of the defect for different activation enthalpies $E_{\mathrm{ann}}$. ( $E_{\mathrm{ann}}=1.0 \mathrm{eV}$ corresponds to the annealing rate reported in Ref. [17].) The solid line is the calculated (Eq. (2)) temperature dependence of the thermal emission rate $e_{n}^{\text {th }}(T)$ for a typical deep level with an activation enthalpy of $E_{\mathrm{a}}=300 \mathrm{meV}$. Electronic defect states can only be measured by thermal space charge spectroscopic methods if a temperature range exists in which the annealing rate of the defect is low, in particular $e_{\text {ann }}$ is in the stable region (hatched area), and $e_{\mathrm{n}}^{\text {th }}$ can be conveniently measured (gray area). In this example the defect would be invisible for $E_{\mathrm{ann}}<0.4 \mathrm{eV}$.

is given by the bold solid line in Fig. 1. The set of dashed curves in the diagram represents $e_{\text {ann }}(T)$ for different $E_{\text {ann }}$. For $E_{\text {ann }}<0.4 \mathrm{eV}$ there is obviously no chance to investigate the defect since for temperatures where thermal electron emission is possible, $e_{\text {ann }}$ is too high such that the defect anneals before the experiment can be accomplished. For $E_{\text {ann }} \approx 0.7 \mathrm{eV}$ there is a temperature range, $150-270 \mathrm{~K}$ in which the defect is stable and can be thermally ionised. If $E_{\text {ann }}>1.0 \mathrm{eV}$ the defect would be considered stable.

From Fig. 1, it is clear that the investigation of unstable defects requires a setup in which the defect can be introduced into the semiconductor at low temperatures such that it will not anneal out. Furthermore the same setup must allow for in situ characterisation of the defect by thermal space charge spectroscopy so that the sample is not exposed to room-temperature for re-mounting.

However, even such a setup is not capable to investigate defects where $e_{\text {ann }}>e_{\mathrm{n}}^{\text {th }}$ holds for all temperatures. A way out of this dilemma is to photo-ionise the unstable defect instead of thermally emitting the charge carrier. The photo-ionisation rate is in first approximation not temperature-dependent and is given by

$$
e_{\mathrm{n}}^{\mathrm{o}}=\sigma_{\mathrm{n}}^{\mathrm{o}}(h \nu) \Phi(h \nu),
$$

where $\sigma_{\mathrm{n}}^{\circ}(h v)$ is the photo-ionisation cross-section which depends on the energy of the incident photons $h v . \Phi(h v)$ is the flux of monochromatic photons of energy $h v$ which is given by the light source. Adding optical excitation to space charge spectroscopy has the advantage that electronic defect 

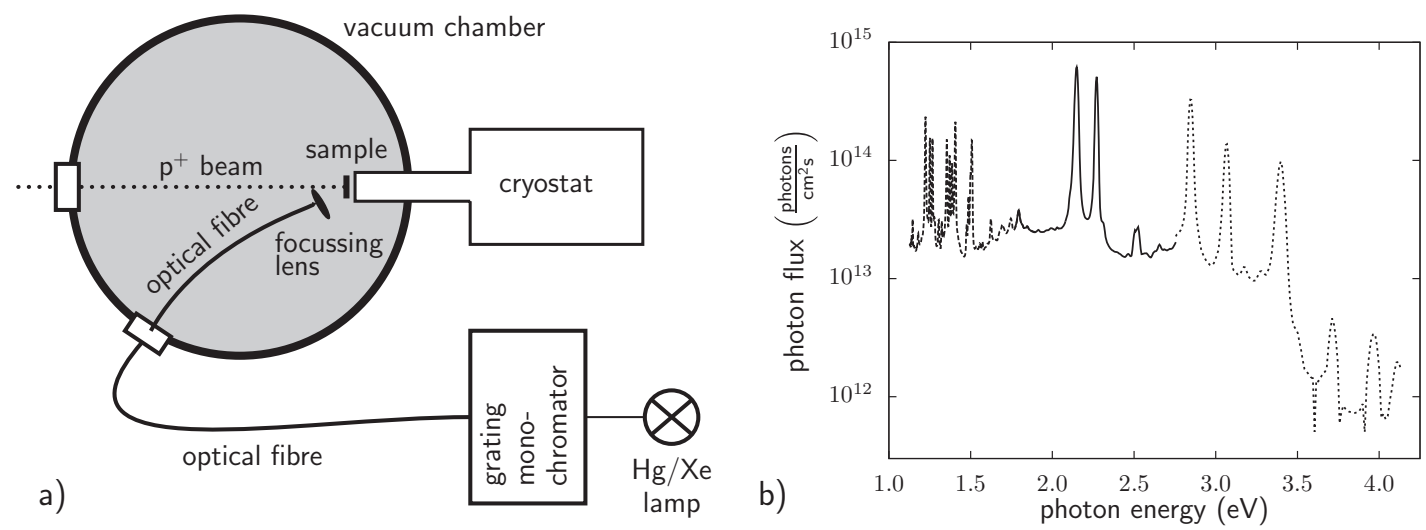

Figure 2 (a) Sketch of the setup. (b) Spectrum of the $\mathrm{Hg} / \mathrm{Xe}$ lamp including the filters at the exit slit of the monochromator, both optical fibers and the focusing lens. (Figure adapted from Ref. [22].)

states can be investigated at any temperature ${ }^{1}$ and therefore a temperature where the defect is stable can be chosen. Furthermore, depending on the optical equipment, in principle the whole bandgap can be scanned for defect states.

In this light we investigated (primary) lattice defects in $\mathrm{GaN}$ thin films that were introduced by proton-bombardment at $20 \mathrm{~K}$. We recently built a setup for this purpose [22] in which the proton-bombardment as well as optical space charge spectroscopy can be conducted and thus introduction and annealing of lattice defects can be investigated in situ.

\section{Experimental}

2.1 Samples The silicon doped GaN thin film sample used in this study was grown by metal-organic chemical vapor deposition onto a 2 inch sapphire substrate, provided by AIXTRON SE (Germany). The thickness of the film was $3.4 \mathrm{um}$, obtained from spectral optical transmission measurements. The substrate was cut into approximately $5 \times 3 \mathrm{~mm}^{2}$ pieces which were degreased, cleaned and etched using the recipe suggested in Ref. [23]. For the fabrication of the ohmic contacts a $\mathrm{Ti} / \mathrm{Al} / \mathrm{Ni} / \mathrm{Au}$ structure was deposited and thermally annealed [23] which resulted in a contact resistance of $1 \mathrm{k} \Omega$. Subsequently $15 \mathrm{~nm}$-thick, semi-transparent Palladium Schottky contacts were resistively evaporated. The electrical connection was realised by thin gold wires bonded to the ohmic as well as to the Schottky contacts by a conductive silver epoxy. In total three samples, in the following referred to as S1, S2, and S3, were fabricated by this method.

2.2 Setup Figure 2a displays a sketch of the setup we used in this study. A van-de-Graaff accelerator provided a mono-energetic proton beam which was focused and guided into a vacuum chamber. In the chamber, the sample was mounted on the cold-finger of a closed-cycle helium cryostat such that the Schottky contact was aligned with the proton beam. Using thin conductive epoxy-bonded gold wires the Schottky and the ohmic contacts were contacted and the capacitance of the sample was measured by an Agilent 4284A

${ }^{1}$ Provided $e_{\mathrm{n}}^{\mathrm{o}} \gg e_{\mathrm{n}}^{\text {th }}$ holds.
LCR meter. The optical setup consisted of a $1000 \mathrm{~W} \mathrm{Hg} / \mathrm{Xe}$ arc lamp, a grating monochromator, an optical fiber through which the light was passed into the vacuum chamber and a lens focusing the light onto the Schottky contact. The photon flux spectrum is shown in Fig. 2b. The setup was therefore suitable to photoemit defect-bound electrons into the $\mathrm{CB}$ from defect states in the vicinity of the valence band (VB) edge up to those approximately $1 \mathrm{eV}$ below the $\mathrm{CB}$ edge. Details of the setup are published in Ref. [22].

2.3 Defect studies From capacitance-voltage $(C-V)$ measurements the net doping density [24] of the as-grown samples S1, S2, and S3 was found to be homogeneous and amounted to $N_{\text {net }}=1.7 \times 10^{17} \mathrm{~cm}^{-3}$. This agrees well with the intentional silicon doping of $N_{\mathrm{Si}} \approx 2 \times 10^{17} \mathrm{~cm}^{-3}$. Photo-capacitance measurements and optical $C-V(\mathrm{OCV})$ measurements [25] at a photon energy of $h v=3.0 \mathrm{eV}$ revealed the presence of a midgap state approximately $1 \mathrm{eV}$ above the VB edge. This level is probably identical with the one previously reported by Calleja et al. [26]. Its concentration was determined as $3 \times 10^{15} \mathrm{~cm}^{-3}$. Furthermore the acceptor concentration was determined as $N_{\mathrm{a}} \approx 1.3 \times$ $10^{16} \mathrm{~cm}^{-3}$ by OCV using a photon energy of $h v=3.4 \mathrm{eV}$.

After this pre-characterisation, all samples underwent the following treatment: (i) cooling to $20 \mathrm{~K}$, (ii) $1.6 \mathrm{MeV}$ proton $\left(\mathrm{p}^{+}\right)$-bombardment at a fluence of $\approx 10^{14} \mathrm{~cm}^{-2}$, (iii) slowly heating to $330 \mathrm{~K}$, and (iv) cooling to $20 \mathrm{~K}$. During steps 1,3 , and 4 capacitance transient experiments were conducted: In steps of $1 \mathrm{~K}$, a $10 \mathrm{~s}$ filling pulse was applied to the sample and afterwards the capacitance was measured for $180 \mathrm{~s}$. The filling pulse was either a voltage pulse of $0.8 \mathrm{~V}$ in forward direction which flattened the bands or the sample was kept under reverse bias but illuminated with monochromatic light of photon energy $h v=3.45 \mathrm{eV}$. During the filling pulses the defect states became occupied by their attributed charge carriers. Subsequently a reverse voltage of $4 \mathrm{~V}$ was applied and the capacitance transient stemming from the emission of defect-bound charge carriers was recorded (sampling rate $11 \mathrm{~Hz}$ ) at a probing voltage of $100 \mathrm{mV}$ and a frequency of $100 \mathrm{kHz}$. The capacitance transients were then 

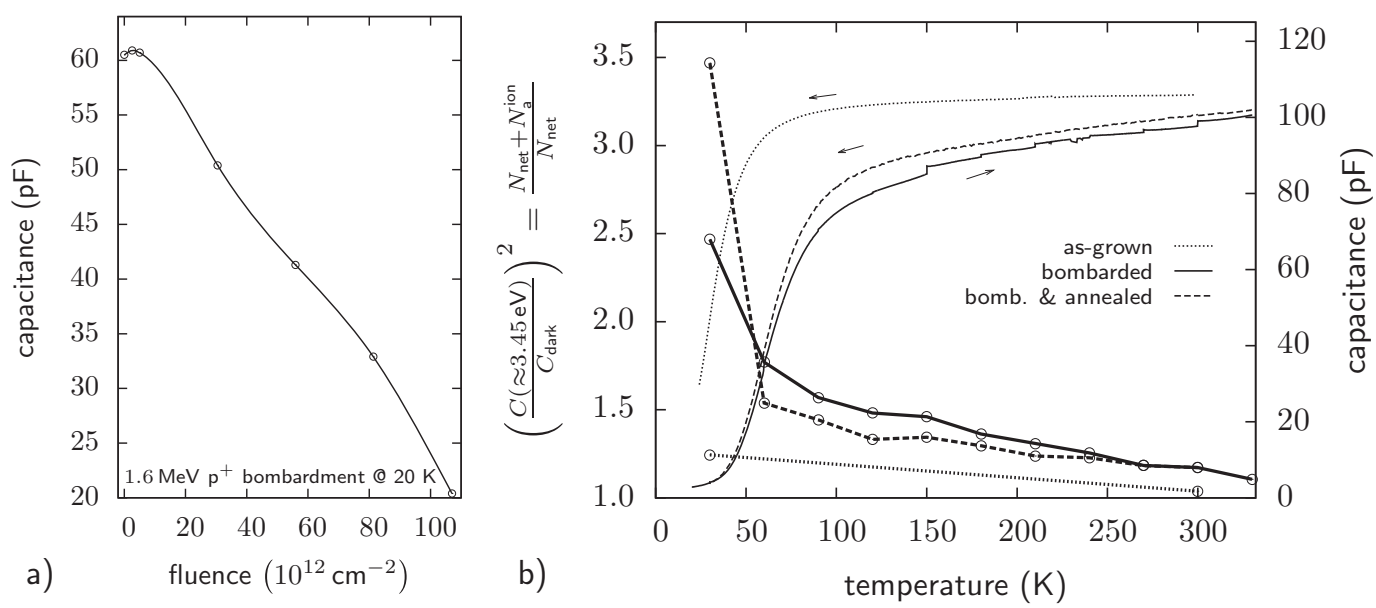

Figure 3 (a) Quasi in situ measurement of the sample capacitance in dependence of the $1.6 \mathrm{MeV}$ proton fluence. (b) Thin lines: capacitancetemperature measurements during the $\mathrm{p}^{+}$-bombardment and annealing cycle. Thick lines and points: amplitude of the normalised squared photo-capacitance peak $(h v \approx 3.4 \mathrm{eV})$ data which is a measure for the concentration of compensating acceptors. The sample was reverse biased at $4 \mathrm{~V}$ and the probing frequency was $100 \mathrm{kHz}$ in both measurements.

digitally processed and the capacitance-temperature $(C-T)$ characteristics and the deep-level transient spectroscopy (DLTS) spectra of the samples were obtained. A further processing of the DLTS data yielded the thermal emission rates of electronic defect states.

In the case of sample $\mathrm{S} 1$, photo-capacitance transients were recorded at constant temperature for different photon energies. As in the thermal transient measurements, a filling pulse of $10 \mathrm{~s}$ length and $0.8 \mathrm{~V}$ forward bias ensured the defect states in the sample to be initially filled. Afterwards a reverse bias of $4 \mathrm{~V}$ was applied, the monochromator shutters were opened and the capacitance was recorded for $360 \mathrm{~s}$. For photon energies $h v$ suitable to ionise electronic states in the vicinity of the VB edge the sample capacitance increased. Assuming that the majority of these states are acceptors, the acceptor compensation degree can be obtained [25] from the capacitance change ${ }^{2}$

$$
\frac{N_{\text {net }}+N_{\mathrm{a}}^{\text {ion }}}{N_{\text {net }}}=\left(\frac{C_{\infty}(h v)}{C_{\infty}(\text { dark })}\right)^{2}
$$

$C_{\infty}$ (dark) is the equilibrium capacitance without illumination, $C_{\infty}(h v)$ is the capacitance under illumination and $N_{\mathrm{a}}^{\text {ion }}$ is the concentration of photo-ionised acceptors.

Using this method, the photo-capacitance of sample S1 was measured at $300 \mathrm{~K}$ and the acceptor compensation degree was determined, see Fig. 3b. Afterwards the sample was cooled to $30 \mathrm{~K}$ and a second photo-capacitance spectrum was recorded. From this spectrum the determined acceptor compensation degree was a bit higher than at $300 \mathrm{~K}$. This can be explained by high thermal hole emission rates of some of the photo-ionised acceptor states at $300 \mathrm{~K}$ making the states invisible in the photo-capacitance spectrum. After sample S1 was further cooled to $20 \mathrm{~K}$ the $\mathrm{p}^{+}$-bombardment was carried

\footnotetext{
${ }^{2}$ In principle for $t \rightarrow \infty$, here at the end of the transient.
}

out in six steps. After each bombardment the capacitance of the sample was measured (frequency ${ }^{3} 10 \mathrm{kHz}, 4 \mathrm{~V}$ reverse bias). The results of these quasi in situ measurements are displayed in Fig. 3a. Thereby the capacitance is related to the free electron concentration. It is remarkable that proton fluences $<5 \times 10^{12} \mathrm{~cm}^{-2}$ cause an increase in the capacitance and therefore in the electron concentration. ${ }^{4} \mathrm{~A}$ further increase of the fluence resulted in a decrease of the capacitance. This can be explained by the drop of the Fermi level caused by the introduction of Frenkel pairs.

After the bombardment, the temperature was slowly increased and, starting at $30 \mathrm{~K}$, photo-capacitance transients were recorded every $30 \mathrm{~K}$. In Fig. $3 \mathrm{~b}$, the $C-T$ data as well as $\left(C_{\infty}(3.45 \mathrm{eV}) / C_{\infty}(\text { dark })\right)^{2}$, which is a measure for photo-ionised shallow acceptors, are displayed for the whole process. The $\mathrm{p}^{+}$-bombardment increased the freeze-out temperature of the sample, an effect that persisted even after the sample was annealed at $330 \mathrm{~K}$. This can be explained by the introduction of thermally stable nitrogen vacancies $\mathrm{V}_{\mathrm{N}}$. Look et al. [7] determined their ionisation energy to $70 \mathrm{meV}$ which is higher than that of the $\mathrm{Si}_{\mathrm{Ga}}$ donor $(26 \mathrm{meV}$, [27]). After the bombardment the concentration of $\mathrm{V}_{\mathrm{N}}$ (and thus also that of interstitial nitrogen acceptors) is in the same order of magnitude as the concentration of the silicon donors. In turn the Fermi level is lower than in the as-grown sample at low temperatures. This directly translates into a lower electron concentration and a higher temperature for charge carrier freeze-out.

\footnotetext{
${ }^{3}$ For the $20 \mathrm{~K}$ measurements the probing frequency was chosen $10 \mathrm{kHz}$ instead of the otherwise used $100 \mathrm{kHz}$ because of the remarkable increase of the series resistance at low temperatures. Ten kilohertz were found to be below the cut-off frequency of the diode such that the capacitance could be reliably measured.

${ }^{4}$ This effect is definitely no artefact as it was reproduced at a different contact of the same sample and the observed change in the capacitance is greater than the noise level of the system.
} 


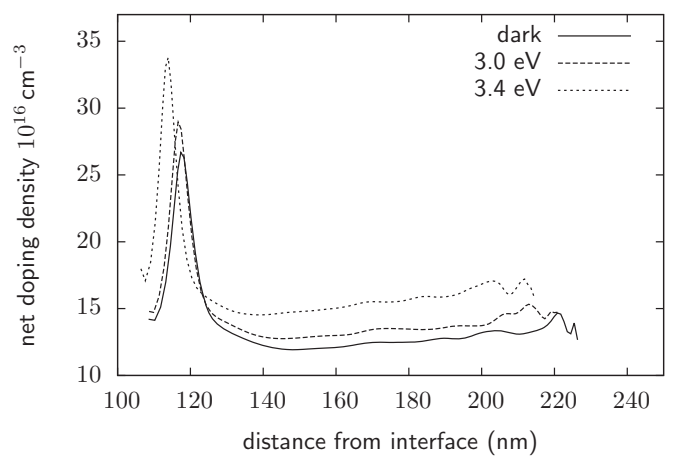

Figure 4 Net doping density in the $\mathrm{p}^{+}$-bombarded sample measured by optical capacitance-voltage spectroscopy at roomtemperature. The oscillator frequency was $100 \mathrm{kHz}$ and the reverse voltage range was $-1 \mathrm{~V}<V<4 \mathrm{~V}$. The peak corresponds to bombardment-induced surface states.

The steps ${ }^{5}$ in the $C-T$ curve of the bombarded sample in combination with the drop in the acceptor compensation degree (solid lines) indicate that either bombardment induced acceptors anneal out or donors are formed by defect reactions at these temperatures. From the step at $\approx 150 \mathrm{~K}$, we deduce that approximately $5 \%$ of the defects in the asbombarded sample anneal and hence a significant proportion of defect reactions observed in this study occur at this temperature. At $T>270 \mathrm{~K}$, the acceptor compensation degree in the bombarded sample before and after annealing (solid lines and dashed lines, respectively) are identical whereas the $C-T$ characteristics still differ and defect annealing is still observed in the as-bombarded sample. This can best be explained by existing donors reacting, forming shallower donors which ionise immediately. The squared normalised photo-capacitance at $30 \mathrm{~K}$ for the annealed sample was almost double that of the un-annealed sample, suggesting that during the annealing stable acceptors were formed whose electronic states are deeper than those of the unstable primary acceptors. The shallow primary states would then be invisible because they immediately emit the photo-generated hole into the VB. This would explain the higher concentration of photo-ionised acceptors after annealing compared to directly after the $\mathrm{p}^{+}$-bombardment.

Finally $C-V$ and OCV measurements where conducted at $300 \mathrm{~K}$, Fig. 4 . From the $C-V$ measurement conducted in the dark the net doping density was found to be homogeneous and amounted to $N_{\text {net }}=1.2 \times 10^{17} \mathrm{~cm}^{-3}$. The peak in the doping profile was found to originate from surface states approximately $1 \mathrm{eV}$ below the $\mathrm{CB}$ edge which were introduced by the $\mathrm{p}^{+}$-bombardment. From the theory of OCV [25] it is known that the difference in the net doping densities measured in the dark and under illumination is equal to the concentration of the photo-ionised defect states. Using photon energies of $h v=3 \mathrm{eV}$, the concentration of the midgap state was determined to $N \approx 10^{16} \mathrm{~cm}^{-3}$ and was therefore higher than in

${ }^{5}$ The primary defect reactions occurred during the photo-capacitance measurements which lasted for $8 \mathrm{~h}$.

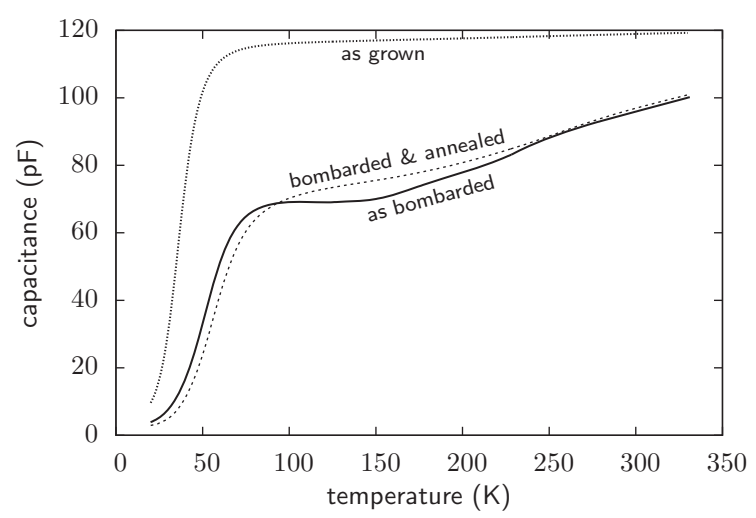

Figure 5 Capacitance-temperature characteristics for the bombardment-annealing cycle of sample S2. The probing frequency was $100 \mathrm{kHz}$ and the reverse voltage was $4 \mathrm{~V}$.

the as-grown sample. OCV measured at $3.4 \mathrm{eV}$ revealed a concentration of photo-ionised acceptors of $3 \times 10^{16} \mathrm{~cm}^{-3}$.

The $\mathrm{p}^{+}$-bombardment-annealing cycle was repeated a second time with another fragment of the GaN thin film, sample S2, but with no optical excitation. This was done in order to avoid that the annealing rate of primary defects is affected by the illumination, for instance because of the different charge state of primary defect due to photo-ionisation. In Fig. 5, the capacitance-temperature characteristics of sample $\mathrm{S} 2$ for the as-grown state, after $\mathrm{p}^{+}$-bombardment and after bombardment and exposure to $330 \mathrm{~K}$ are displayed. As expected, the $C-T$ characteristics of the as-grown sample does not significantly differ from the one measured on S1. However, after $\mathrm{p}^{+}$-bombardment the $C-T$ characteristics of S1 and S2 are different. Obviously directly after the $\mathrm{p}^{+}$-bombardment the concentration of compensating acceptors in sample S2 was higher than in S1 as can be deduced from the lower "freeze-out" step in the $C-T$ characteristics at $\approx 60 \mathrm{~K}$. This might be due to "photo"-annealing of acceptors in the case of sample S1. It could also originate from slightly different shading of the contacts by the epoxy blobs which were used for bonding the gold wires to the Schottky contacts.

The difference in the capacitances of sample S2 directly after the $\mathrm{p}^{+}$-bombardment and after annealing the bombarded sample hints to a significant annealing of $\mathrm{p}^{+}$-bombardmentinduced acceptor states already at approximately $150 \mathrm{~K}$ as was also observed in the experiments on sample S1. For $T>$ $250 \mathrm{~K}$ the $C-T$ curves are equal meaning that from thereon until $330 \mathrm{~K}$ no more annealing occurred in this sample.

The digital correlation of the capacitance transients with the correlation function used by Ellguth et al. [28] yielded the DLTS spectra shown in Fig. 6a. Only one deep-level was found in the spectrum of the as-grown sample, see inset of DLTS spectrum in Fig. 6a. From standard Arrhenius analysis of $e_{\mathrm{n}}^{\text {th }}(T)$ [18], see Fig. 6b, thermal activation enthalpy and high temperature limit of the electron capture cross-section of this level were determined to $E_{\mathrm{a}} \approx 490 \mathrm{meV}$ and $\sigma_{\mathrm{n}}^{\infty}=2.5 \times$ $10^{-17} \mathrm{~cm}^{2}$. This deep-level is most likely the $\mathrm{E} 1$ state reported 

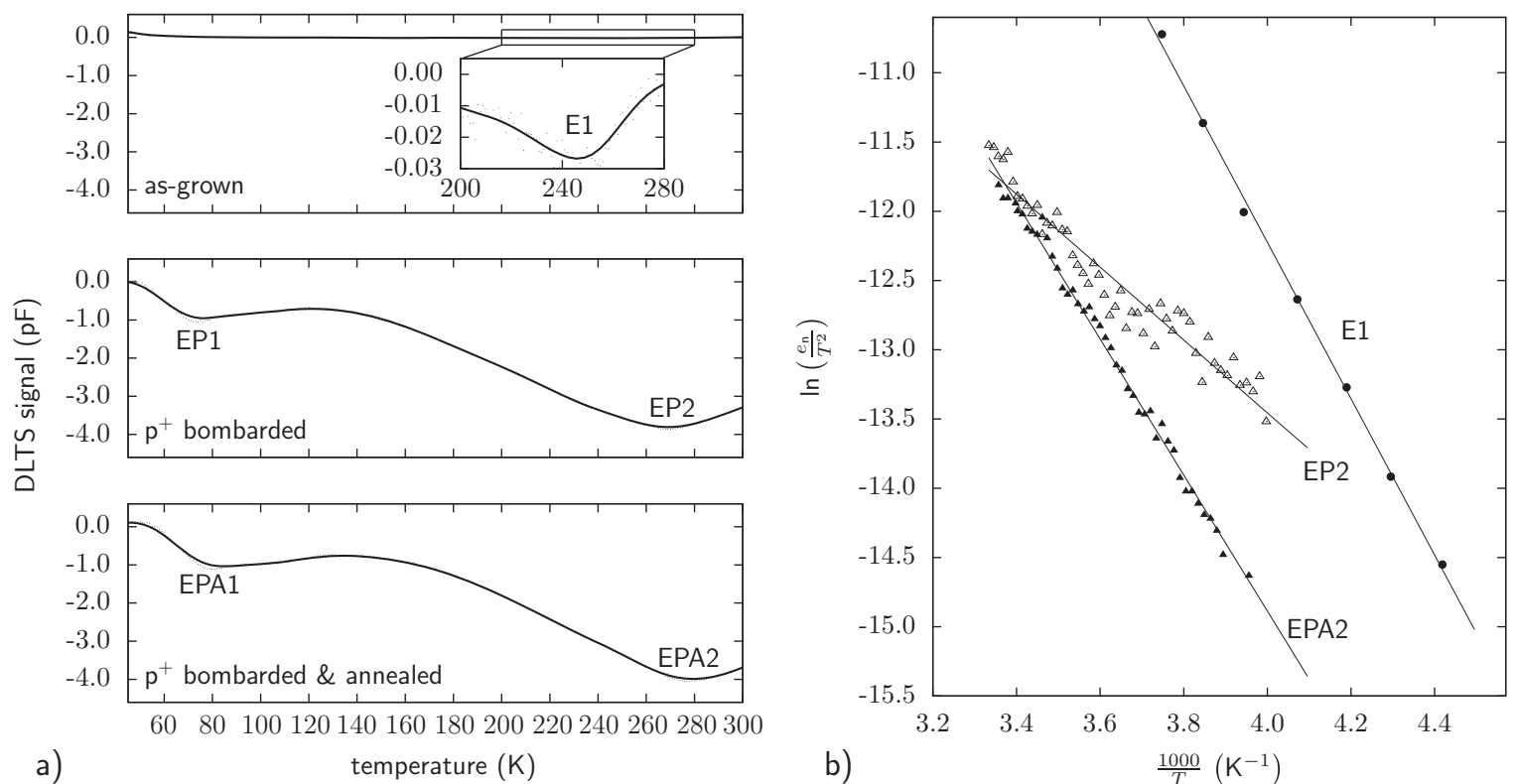

a)

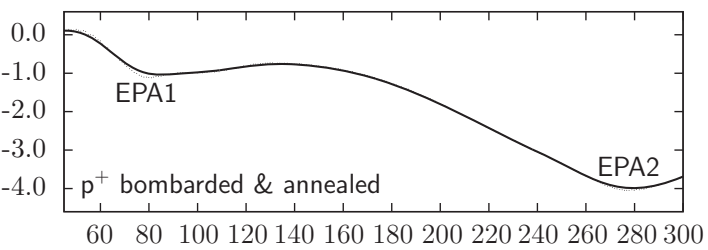

temperature $(\mathrm{K})$

b)

Figure 6 (a) DLTS spectra recorded on sample S2 using $10 \mathrm{~s}$ forward voltage pulses that almost flattened the bands. The reverse bias during the recording of the capacitance transient amounted to $4 \mathrm{~V}$ and the rate window frequency was $1 / 32 \mathrm{~Hz}$. The filling pulse was $0.8 \mathrm{~V}$ in forward direction for $10 \mathrm{~s}$. (b) Arrhenius plot of $e_{\mathrm{n}}^{\text {th }}(T)$ of the deep-levels E1, EP2, and EPA2.

by Götz et al. [29], which commonly occurs in metal-organic chemical vapor deposition grown $\mathrm{GaN}$.

After the $\mathrm{p}^{+}$-bombardment majority carrier emission from defects resulted in a negative DLTS signal in the whole temperature range (second spectrum in Fig. 6a). Thereby DLTS peaks of at least two deep-levels, EP1 and EP2, were found. In addition the spectrum is superimposed on a signal stemming from non-exponential transients that increases slightly with increasing temperature. Most-likely this is due to the emission of electrons from bombardment-induced surface states, see Fig. 4. The DLTS peaks of the deep-levels EP1 and EP2 can be evaluated by Arrhenius analysis of $e_{\mathrm{n}}^{\text {th }}(T)$ resulting in:

\begin{tabular}{lll}
\hline & $E_{\mathrm{a}}(\mathrm{meV})$ & $\sigma_{\mathrm{n}}^{\infty}\left(\mathrm{cm}^{2}\right)$ \\
\hline EP1 & 200 & $3 \times 10^{-13}$ \\
EP2 & 225 & $4 \times 10^{-23}$ \\
\hline
\end{tabular}

For the sake of completeness we remark that the EP1 DLTS peak exhibits a shoulder on the high-temperature side, probably stemming from another deep-level.

On the first glance the DLTS spectrum of the bombarded sample did not change significantly after the sample was exposed to room-temperature (Fig. 6a). As directly after the $\mathrm{p}^{+}$-bombardment two deep-level peaks, in the following labelled EPA1 and EPA2, were found. Arrhenius analysis yielded:

\begin{tabular}{lll}
\hline & $E_{\mathrm{a}}(\mathrm{meV})$ & $\sigma_{\mathrm{n}}^{\infty}\left(\mathrm{cm}^{2}\right)$ \\
\hline EPA1 & 225 & $8 \times 10^{-13}$ \\
EPA2 & 425 & $1 \times 10^{-19}$ \\
\hline
\end{tabular}

It is reasonable to assume EP1 and EPA1 as well as EP2 and EPA2 to be the same defects but in a different environment. In both cases $E_{\mathrm{a}}$ is lower and $\sigma_{\mathrm{n}}^{\infty}$ is smaller after the irradiation. Whereas this difference is marginal for EP1/EPA1 it is remarkable for EP2/EPA2 and manifests in the Arrhenius plots of $e_{\mathrm{n}}^{\text {th }}(T)$ displayed in Fig. 6b. Most likely this observation can be explained by the high defect density in the as-bombarded sample whereas it was lower after the sample was exposed to $330 \mathrm{~K}$. In the first case, there will be a stronger overlap of defect orbitals, which is known to lead to lower defect ionisation energies but also smaller capture cross-sections. Especially the presence of a high acceptor density, as was found directly after the $\mathrm{p}^{+}$-bombardment, could account for this effect. The Arrhenius plots of EP1 and EPA1 are similar to those of the ER3 deep level reported by Goodman et al. [6]. There ER3 was found to be introduced by high-energy electron irradiation as well as by proton or alpha-particle bombardment.

A third sample, S3, underwent the same bombardmentannealing cycle but the capacitance transient measurement was conducted using $3.45 \mathrm{eV}$ optical pulses instead of a forward bias. In doing so electron-hole pairs were generated in the space charge region during the pulse and empty ${ }^{6}$ hole traps close to the VB became occupied by photo-emitting a bound electron into the CB. The capacitance-temperature measurements for the cycle under these conditions are displayed in Fig. 7. A major difference to the $C-T$ cycles measured on the samples S1 and S2 is that in this case, because of the UV pulses, most of the acceptor states in the vicinity of the VB were photo-ionised. Therefore the

\footnotetext{
${ }^{6} \mathrm{Can}$ also be seen as occupied by an electron.
} 


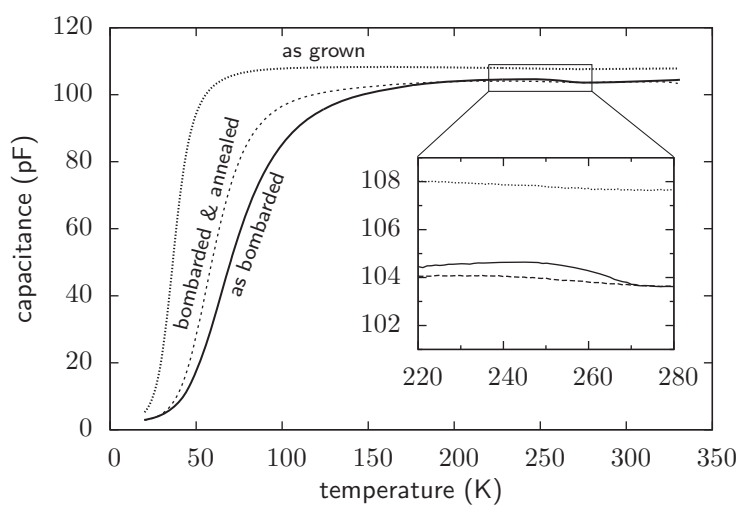

Figure 7 Capacitance-temperature characteristics for the bombardment-annealing cycle of sample S3. The probing frequency was $100 \mathrm{kHz}$ and the reverse voltage was $4 \mathrm{~V}$. A magnification of the temperature range from 220 to $280 \mathrm{~K}$ (inset) reveals the annealing of an acceptor-like defect which manifests in a drop of $C$ with increasing $T$ for the as-bombarded characteristics.

capacitance is much larger and almost equal to that of the as-grown sample. In other words, the difference in the capacitances measured on sample S2 and S3 (Figs. 5 and 7) reflects the acceptor compensation degree of the samples. A very remarkable observation is that the capacitance of the as-bombarded sample is higher than in the bombarded and annealed sample in the temperature rage from 180 to $270 \mathrm{~K}$. The drop in the capacitance of the as-bombarded sample, see inset of Fig. 7, does not depend on the oscillator frequency and vanished after exposing the sample to $330 \mathrm{~K}$. This is a clear indication that an acceptor state that was photo-ionised by the UV pulses annealed at approximately $260 \mathrm{~K}$.

The DLTS spectra, recorded using optical instead of voltage pulses, are shown in Fig. 8a. The spectrum measured on the as-grown sample showed very little features and the DLTS signal was almost zero in the whole temperature range which is a further indication for the high purity of the GaN mate- rial. However, after the $\mathrm{p}^{+}$-bombardment the DLTS signal became positive meaning that the thermal emission of charge carriers from the hole traps prevailed the electron emission from deep-levels in the vicinity of the CB. Remarkably, only after the sample was exposed to $330 \mathrm{~K}$ the emission of a distinct hole trap manifested as a peak at $\approx 60 \mathrm{~K}$ in the spectrum. In the following this hole trap will be labelled HPA1. Form the temperature dependence of the thermal hole emission rate $e_{\mathrm{p}}^{\text {th }}(T)$ of HPA1 its thermal activation enthalpy and high-temperature limit of the hole capture cross-section ${ }^{7}$ were determined to $E_{\mathrm{a}} \approx 100 \mathrm{meV}$ and $\sigma_{\mathrm{p}}^{\infty} \approx 2 \times 10^{-14} \mathrm{~cm}^{2}$, respectively. The Arrhenius plot of $e_{\mathrm{p}}^{\text {th }}(T)$ is depicted in Fig. $8 \mathrm{~b}$. There is evidence that HPA1 is related to the L1 level observed by ODEPR by Chow et al. [4]. In their study the L1 signal also emerged after the low-temperature electronirradiated sample was exposed to room-temperature and was only observed under 3.41 or $3.53 \mathrm{eV}$ illumination. Chow et al. attributed L1 either to a nitrogen vacancy complex or a gallium vacancy complex.

3 Conclusions We employed thermal and optical space charge spectroscopic measurements to study protonbombardment induced electronic defect states in GaN thin films. The samples were bombarded at $20 \mathrm{~K}$ such that the introduced primary defects did not anneal before they could be studied. Quasi in situ measurements enabled us to monitor the capacitance during the bombardment. In accordance with previous experiments $[5,10]$ a high resilience of $\mathrm{GaN}$ to $1.6 \mathrm{MeV}$ protons even up to a fluence of $10^{14} \mathrm{~cm}^{-3}$ was observed. Photo-capacitance transient measurements allowed for the measurement of the degree of acceptor compensation in the sample. Directly after the bombardment the acceptor concentration was at least a factor of 1.5 higher than the net doping density compared to less than 0.25 in the as-grown sample. After bombardment, the annealing of

${ }^{7}$ Assuming an effective hole mass of $m_{\mathrm{h}}^{\star}=1.25 m_{\mathrm{e}}[30]$.
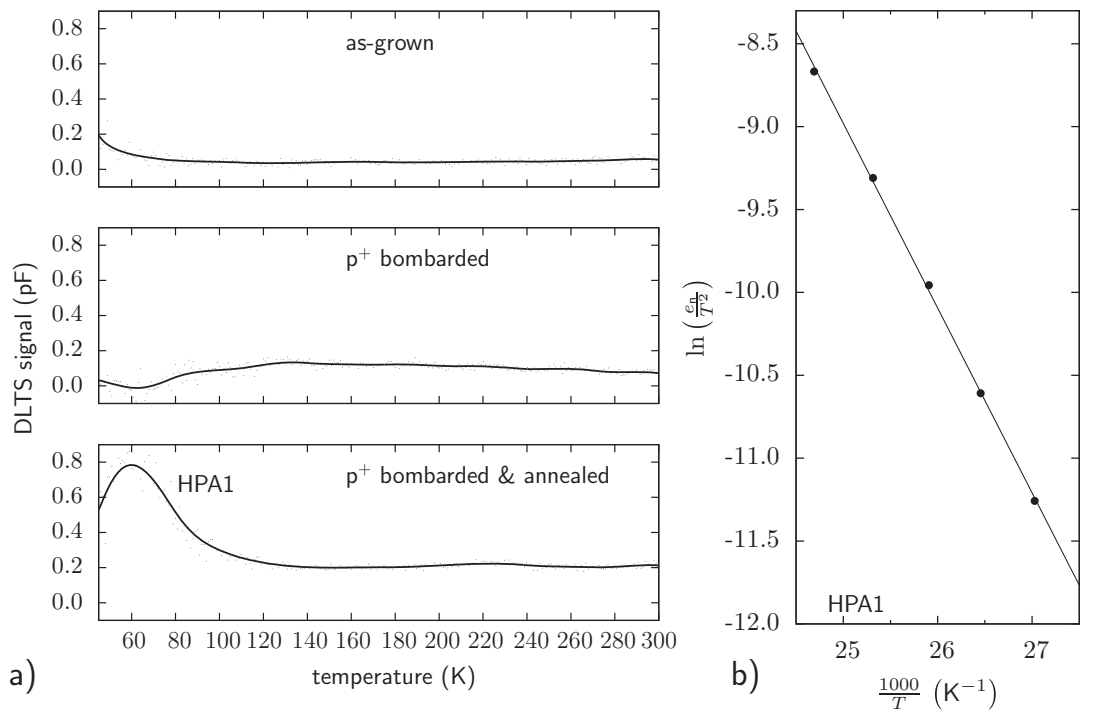

Figure 8 (a) DLTS spectra recorded on sample S3 using optical $(h v=3.45 \mathrm{eV})$ filling pulses instead of voltage pulses. The filling pulse length was $10 \mathrm{~s}$, the reverse bias amounted to $4 \mathrm{~V}$ and the rate window frequency was $1 / 32 \mathrm{~Hz}$. (b) Arrhenius plot of the thermal hole emission rate of the HPA 1 hole trap visible at approximately $60 \mathrm{~K}$ in the DLTS spectrum of the bombarded and annealed sample. 
acceptor states was observed by measuring the capacitance and the acceptor concentration while the temperature was slowly increased. It was concluded that a significant fraction of primary defect reactions occur at $150 \mathrm{~K}$ which could in part account for the radiation hardness of $\mathrm{GaN}$ at roomtemperature.

Conventional deep-level transient spectroscopy revealed the existence of the E1 deep-level in the as-grown material. The low-temperature proton-bombardment introduced two further deep-levels which did not anneal out up to $330 \mathrm{~K}$. However, it was observed that their activation enthalpy and their electron capture cross-sections increased during the annealing. The usage of UV light pulses instead of voltage pulses for the $C-T$ and the DLTS experiments confirmed the high acceptor compensation degree that was observed in the photo-capacitance measurements. Furthermore the annealing of a primary, proton-bombardment induced acceptor state was observed at approximately $260 \mathrm{~K}$. A shallow, stable hole trap, HPA1, was formed in the bombarded sample after the sample was exposed to $330 \mathrm{~K}$. HPA 1 is possibly related to the previously observed L1 ODEPR signal in electron irradiated $\mathrm{GaN}$ after exposure to room-temperature [4].

In summary, we demonstrated that our recently built setup is capable to investigate electronic states of primary, particle-radiation induced lattice defects in wide bandgap semiconductors. Future work will be the determination of annealing rates of these defects and proposing microscopic models describing the experimental findings.

Acknowledgements The authors wish to thank AIXTRON SE (Aachen, Germany), in particular Dirk Fahle, Christian Czekalla, Hannes Behmenburg, and Michael Heuken for providing the GaN thin film sample. We are grateful to the mechanical workshop of the Department of Physics at the University of Pretoria for the fabrication of numerous parts of the setup. We wish to thank two referees for valueable comments that significantly improved the manuscript. Matthias Schmidt was funded by the Postdoctoral Fellowship Program of the University of Pretoria. This work is based upon research supported by the National Research Foundation (NRF). Any opinion, findings and conclusions or recommendations expressed in this material are those of the author(s) and therefore the NRF does not accept any liability in regard thereto.

\section{References}

[1] H. Morkoc, Handbook of Nitride Semiconductor Devices, Vol. 1, first ed. (Wiley-VCH Verlag, Weinheim, 2008).

[2] C. Bozdog, H. Przybylinska, G. D. Watkins, V. Härle, F. Scholz, M. Mayer, M. Kamp, R. J. Molnar, A. E. Wickenden, D. D. Koleske, and R. L. Henry, Phys. Rev. B 59, 12479-12486 (1999).

[3] K. H. Chow, G. D. Watkins, A. Usui, and M. Mizuta, Phys. Rev. Lett. 85, 2761 (2000).

[4] K. H. Chow, L. S. Vlasenko, P. Johannesen, C. Bozdog, G. D. Watkins, A. Usui, H. Sunakawa, C. Sasaoka, and M. Mizuta, Phys. Rev. B 69, 045207 (2004).
[5] S. M. Khanna, J. Webb, H. Tang, A. J. Houdayer, and C. Carlone, IEEE Trans. Nucl. Sci. 47, 2322-2328 (2000).

[6] S. A. Goodman, F. D. Auret, F. K. Koschnick, J.-M. Spaeth, B. Beaumont, and P. Gibart, Mater. Sci. Eng. B 71, 100-103 (2000).

[7] D. C. Look, G. C. Farlow, P. J. Drevinsky, D. F. Bliss, and J. R. Sizelove, Appl. Phys. Lett. 17, 3525 (2003).

[8] F. Tuomisto, V. Ranki, D. C. Look, and G. C. Farlow, Phys. Rev. B 76, 165207 (2007).

[9] H.-Y. Kim, J. Ahn, M. A. Mastro, C. R. Eddy, Jr., et al., J. Vac. Sci. Technol. B 27, L11-L13 (2009).

[10] B. D. White, M. Bataiev, S. H. Goss, X. Hu, A. Karmarkar, D. M. Fleetwood, R. D. Schrimpf, W. J. Schaff, and L. J. Brillson, IEEE Trans. Nucl. Sci. 50, 1791-1796 (2003).

[11] A. Kalavagunta, M. Silvestri, M. J. Beck, S. K. Dixit, and R. D. Schrimpf, IEEE Trans. Nucl. Sci. 56, 3192-3195 (2009).

[12] A. Y. Polyakov, S. J. Pearton, P. Frenzen, F. Ren, L. Liu, and J. Kim, J. Mater. Chem. C 1, 877-887 (2013).

[13] A. Ionascut-Nedelcescu, C. Carlone, A. Houdayer, H. J. von Bardeleben, J.-L. Cantin, and S. Raymond, IEEE Trans. Nucl. Sci. 49, 2733-2738 (2002).

[14] J. Nord, K. Nordlund, and J. Keinonen, Phys. Rev. B 68, 184104 (2003).

[15] D. Pons, P. M. Mooney, and J. C. Bourgoin, J. Appl. Phys. 51, 2038-2042 (1980).

[16] D. V. Lang, and L. C. Kimling, Phys. Rev. Lett. 33, 489-492 (1974).

[17] D. V. Lang, L. C. Kimerling, and S. Y. Leung, J. Appl. Phys. 47, 3587-3591 (1976).

[18] P. Blood and J. W. Orton, The Electrical Characterization of Semiconductors: Majority Carriers and Electron States, Vol. 1 (Academic Press, US, 1992).

[19] G. L. Pearson and J. Bardeen, Phys. Rev. 75, 865-883 (1949).

[20] M. Schmidt, M. Ellguth, R. Karsthof, H. V. Wenckstern, R. Pickenhain, M. Grundmann, G. Brauer, and F. C. C. Ling, Phys. Status Solidi B 249, 588-595 (2012).

[21] J. D. Weeks, J. C. Tully, and L. C. Kimerling, Phys. Rev. B 12, 3286-3292 (1975).

[22] M. Schmidt, J. Janse van Rensburg, H. de Meyer, W. E. Meyer, F. D. Auret, F. Schmidt, and H. von Wenckstern, in: Proceedings of SAIP2012, the 57th Annual Conference of the South African Institute of Physics, under review. Available online at http: www.saip.org.za

[23] J. Janse van Rensburg, F. D. Auret, V. S. Matias, and A. Vantomme, Physica B 404, 4411-4414 (2009).

[24] C. van Opdorp, Solid-State Electron. 11, 397-406 (1968).

[25] M. Schmidt, H. V. Wenckstern, R. Pickenhain, and M. Grundmann, Solid-State Electron. 75, 48-54 (2012).

[26] E. Calleja, F. J. Sánchez, D. Basak, et al., Phys. Rev. B 55, 4689-4694 (1997).

[27] A. E. Wickenden, L. B. Rowland, K. Doverspike, D. K. Gaskill, J. A. Freitas, Jr., D. S. Simons, and P. H. Chi, J. Electron. Mater. 24, 1547-1550 (1995).

[28] M. Ellguth, M. Schmidt, R. Pickenhain, H. von Wenckstern, and M. Grundmann, Phys. Status Solidi 248, 941-949 (2011).

[29] W. Götz, N. M. Johnson, H. Amano, and I. Akasaki, Appl. Phys. Lett. 65, 463-465 (1994).

[30] B. Săntić, Semicond. Sci. Technol. 18, 219-224 (2003). 